\title{
Monte Carlo simulation for statistical Hauser-Feshbach theory
}

\author{
T. Kawano ${ }^{1, a}$, P. Talou, M. B. Chadwick, and T. Watanabe \\ Theoretical Division, Los Alamos National Laboratory, Los Alamos, NM 87545, U.S.A.
}

\begin{abstract}
Monte Carlo simulations for particle and $\gamma$-ray emissions from a compound nucleus based on the Hauser-Feshbach statistical theory are performed. The Monte Carlo method is applied to the neutron induced nuclear reactions on ${ }^{56} \mathrm{Fe}$, and the results are compared with a traditional deterministic method. The neutron and $\gamma$-ray emission correlation is examined by gating on an $847 \mathrm{keV} \gamma$-ray that is produced by an inelastic scattering process. The partial $\gamma$-ray energy spectra for different $\gamma$-ray multiplicities is inferred using this Monte Carlo method.
\end{abstract}

\section{Introduction}

Nuclear reaction model codes like GNASH [1], have rather complicated algorithms to generate reaction-wise (or exclusive) energy spectra for individual particle emission process. In the GNASH case, all the decay probabilities at each intermediate stage are recorded, and the data are processed with a supplemental FORTRAN program, RECOIL [2], to calculate the exclusive spectra. A straightforward and more accurate approach to understand all the particle and $\gamma$-ray emission probabilities in the nuclear reaction, is to utilize a Monte Carlo technique. Instead of performing the complicated integration over all the probabilities at each intermediate stage in the course of a compound nucleus decay, we simulate the particle emission process by the Monte Carlo method, and record all the particles and $\gamma$ rays with their energies until the residual nucleus reaches its ground state.

The Monte Carlo technique also facilitates new insights into the nuclear reaction, as correlations between different processes can be studied by gating on a particular $\gamma$-ray so as to provide useful information on spin and parity distributions in the continuum state [3]. Coincidence experiments, i.e. a scattered neutron detection gated by a particular $\gamma$-ray transition, will provide an important benchmark of the modeling of nuclear properties. This kind of investigation, in both theory and experiments, will be more important in the near future for acquiring reliable nuclear data. The Monte Carlo method may help answer questions regarding nuclear reaction mechanisms that have been difficult to address by traditional techniques.

Based on this Monte Carlo study for the Hauser-Feshbach model [4], we can also envision a more rigorous simulation of neutron and $\gamma$-ray emissions from fission fragments. In the past, a Monte Carlo simulation for the prompt fission neutron emission has been performed [5-8], assuming that the neutron emission spectra from the fission fragments follow a Weisskopf probability distribution. Our present

\footnotetext{
a e-mail: kawano@lanl.gov
}

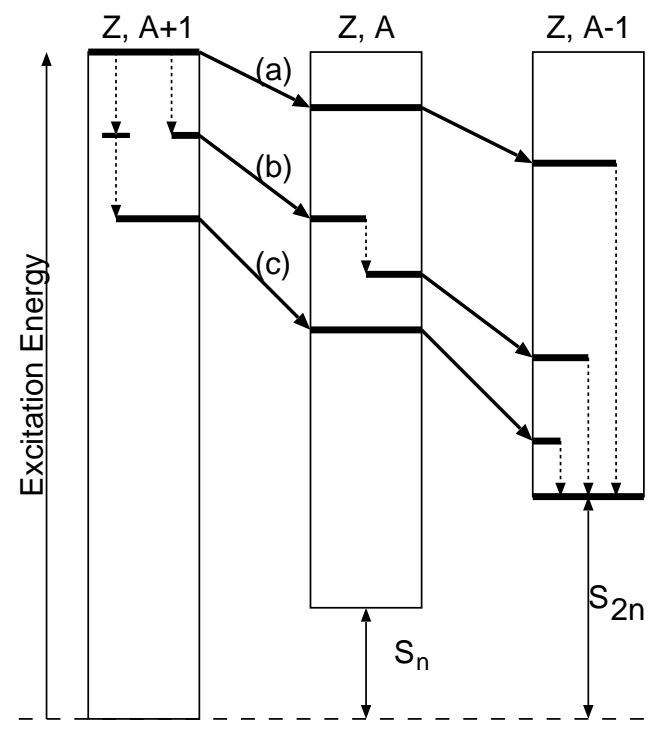

Fig. 1. Schematic picture of multiple neutron emission process from a compound nucleus $A-1$. The solid arrows are the neutron emission, and the dotted arrows are the $\gamma$-ray emission.

work aims at replacing this simple approximation by a more fundamental Hauser-Feshbach treatment for each compound state in the fission fragments.

\section{Calculation Method}

\subsection{Deterministic method for spectrum calculation}

Figure 1 schematically shows sequential neutron emissions from a compound nucleus $(Z, A+1)$, including $\gamma$-ray emissions at each intermediate state. Three paths are depicted as examples, all leading to the ground state of $(Z, A-1)$ nucleus: (a) two neutrons are successively emitted from the initial compound nucleus, and the residual nucleus decays

This is an Open Access article distributed under the terms of the Creative Commons Attribution-Noncommercial License, which permits unrestricted use, distribution, and reproduction in any noncommercial medium, provided the original work is properly cited. 
by emitting a $\gamma$-ray, (b) one $\gamma$-ray emission takes place in both $(Z, A+1)$ and $(Z, A)$ nuclei, and (c) multiple $\gamma$ ray emissions happen in the first compound nucleus. The exclusive neutron emission spectrum for the $(n, 2 n)$ reaction can be calculated by summing up all the probabilities for the possible paths of neutron emission process between $(Z, A+1)$ and $(Z, A-1)$ nuclei.

To describe a formula to calculate the exclusive spectrum, we first need to define a decay probability from a given compound state $\left(c_{n}, k_{n}\right)$, where $c_{n}$ denotes the compound nucleus, $k_{n}$ is the index of excited state with the energy bin width of $\Delta E$, and the suffix $n$ describes the $n$-th compound nucleus. The index $k$ runs from the highest excitation toward the ground state. We define a probability $P\left(c_{n}, k_{n}, c_{m}, k_{m}\right)$, which is a decay probability of the state $\left(c_{n}, k_{n}\right)$ by emitting a particle or a $\gamma$-ray to form a $\left(c_{m}, k_{m}\right)$ state. The probability $P$ can be calculated with the HauserFeshbach theory,

$$
P\left(c_{n}, k_{n}, c_{m}, k_{m}\right)=\frac{T\left(c_{m} k_{m} \rightarrow c_{n} k_{n}\right)}{\sum_{c_{m} k_{m}} T\left(c_{m} k_{m} \rightarrow c_{n} k_{n}\right)},
$$

where $T\left(c_{m} k_{m} \rightarrow c_{n} k_{n}\right)$ is the particle or $\gamma$-ray transmission coefficients from the final $\left(c_{m}, k_{m}\right)$ to initial $\left(c_{n}, k_{n}\right)$ states. Note that all the quantum numbers of $T$ are already summed.

An algorithm to calculate the exclusive spectrum may depend on how much complexity we introduce in evaluating the decay path in Fig 1. Increasing the $\gamma$-ray multiplicities results in more summations over the number of intermediate stages. For example, the path (a) has the multiplicity of one, while it becomes 3 for the cases of (b) and (c). Because the particle emission probabilities are much larger than the $\gamma$-ray emission probability, it is often good enough to consider the path (a) only. However, we allow the $\gamma$-ray competition at each compound nucleus in this study.

\subsection{Monte Carlo technique}

Once the probabilities $P\left(c_{n}, k_{n}, c_{m}, k_{m}\right)$ are constructed with the Hauser-Feshbach formula, a Monte Carlo simulation for the particles and $\gamma$-ray decay processes is straightforward. Starting with the initial state $\left(c_{0}, k_{0}\right)$, a following state can be determined by throwing a dice based on the probability $P\left(c_{0}, k_{0}, c_{m}, k_{m}\right)$, where we know that

$$
\sum_{c_{m} k_{m}} P\left(c_{0}, k_{0}, c_{m}, k_{m}\right)=1 .
$$

We repeat this random sampling procedure until the nucleus reaches a stable state, and record all the particles and $\gamma$-rays emitted during the sequence. In this procedure, since the particle and $\gamma$-ray multiplicities are unlimited, we can reconstruct very accurately the exclusive particle emission spectra by tallying the recorded Monte Carlo histories.

The theoretical model calculations are always in the center-of-mass system (CMS), despite the fact that particle transport calculations require all the quantities in the laboratory system (LAB). The Monte Carlo technique allows

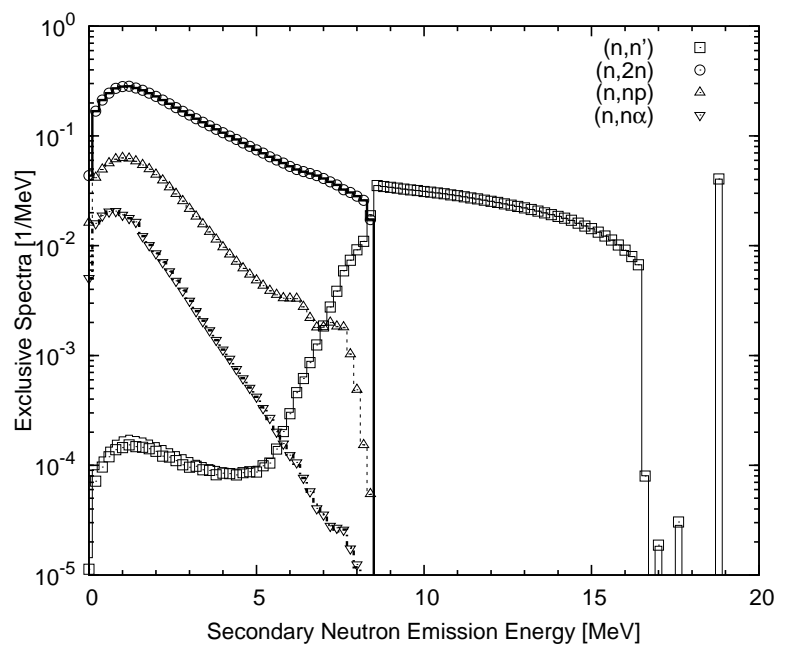

Fig. 2. Exclusive spectra for the (n,n'), (n,2n), (n,np) and (n,n $\alpha)$ reactions calculated with the deterministic method (histograms) and the Monte Carlo method (symbols). The spectra are normalized per outgoing energy per incident neutron.

us to calculate recoils of residual nuclei in the laboratory frame. Although we do not discuss the system conversion between LAB and CMS here, we would like to note that quantities obtained with the Monte Carlo simulation are directly connected to what we see in a real experiment. Hereafter all the particle and $\gamma$-ray energies are in the CMS.

\subsection{Hauser-Feshbach model calculation}

The CoH Hauser-Feshbach code [9] has been upgraded recently. The version 3.0 code, $\mathrm{CoH}_{3}$, calculates all the nuclear reaction cross sections above the resonance range, including the total, shape elastic scattering, direct inelastic scattering, direct/semidirect capture cross sections, preequilibrium emission, and particle and $\gamma$-ray emission in the compound decay process. All the particle transmission coefficients are calculated internally, so that it does not require an external solver of optical model potentials. The code is adopted to generate the probabilities in Eq. (1).

\section{Results and Discussion}

\subsection{Comparison of deterministic and Monte Carlo methods}

As an example, we consider a $20-\mathrm{MeV}$ neutron induced reaction on ${ }^{56} \mathrm{Fe}$. To obtain a satisfactory statistics in the Monte Carlo simulation, we tallied $10^{8}$ histories of particle and $\gamma$-ray decays for the $20-\mathrm{MeV}$ neutron induced reactions on ${ }^{56} \mathrm{Fe}$ case. Figure 2 shows a comparison of the calculated exclusive spectra by both deterministic and Monte Carlo methods for (n,n'), (n,2n), (n,np) and (n,n $\alpha)$ reactions. Each spectrum is normalized per incident neutron per outgoing energy. Therefore

$$
\int \phi_{n^{\prime}}(\epsilon) d \epsilon=\frac{\sigma_{n^{\prime}}}{\sigma_{R}}
$$




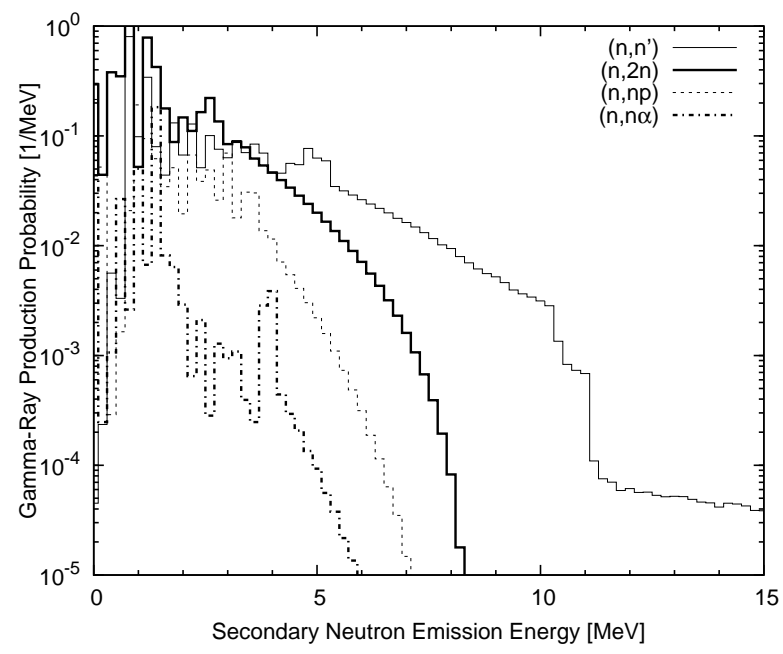

Fig. 3. Exclusive $\gamma$-ray spectra for the (n,n'), (n,2n), (n,np) and $(\mathrm{n}, \mathrm{n} \alpha)$ reactions calculated with the Monte Carlo method. The spectra are normalized per outgoing energy per incident neutron.

and

$$
\int \phi_{2 n}(\epsilon) d \epsilon=\frac{\sigma_{2 n}}{\sigma_{R}},
$$

hold, where $\sigma_{n^{\prime}}$ and $\sigma_{2 n}$ are the inelastic and (n,2n) reaction cross sections, and $\sigma_{R}$ is the total reaction cross section calculated with the optical model. These two reactions are clearly separated at the neutron separation energy, and the two methods give almost identical results, except for tiny differences at low energies of (n.n') spectrum. The agreement for the $(\mathrm{n}, \mathrm{np})$ and $(\mathrm{n}, \mathrm{n} \alpha)$ reaction cases is also excellent. It is not so surprising that these two methods agree very well, as we started with the same probability distributions, together with the fact that the $\gamma$-ray transition probabilities are usually much smaller than the particle emission probabilities.

\section{$3.2 \gamma$-ray emission spectra and multiplicities}

The $\gamma$-ray emission spectra and the $\gamma$-ray multiplicities corresponding to a specific reaction can be obtained very easily with the Monte Carlo technique, by summing all the $\gamma$-rays emitted and dividing by the number of simulations performed, which is shown in Fig. 3. The calculated $\gamma$-ray multiplicities are 2.57 for the (n,n') and 1.67 for $(n, 2 n)$ reactions.

\subsection{Particle and $\gamma$-ray correlation}

One of the notable benefits of the Monte Carlo method is that we can sample any kind of reactions by looking into the history record. As an example, we calculate $20-\mathrm{MeV}$ neutron induced reactions on ${ }^{56} \mathrm{Fe}$ again, and observe an inelastic scattering process that produces an $847 \mathrm{keV} \gamma$-ray, which is a transition from the first $2^{+}$state to the $0^{+}$ground state in ${ }^{56} \mathrm{Fe}$. In this simulation, we are gating on the inelastic scattering process by the partial $847 \mathrm{keV} \gamma$-ray, so

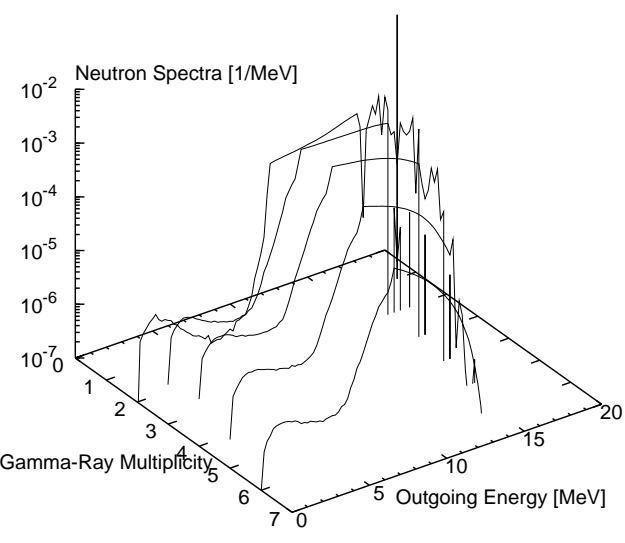

Fig. 4. Inelastic scattering neutron energy spectra from ${ }^{56} \mathrm{Fe}$ by $20-\mathrm{MeV}$ incident neutron. The outgoing neutrons are virtually gated by a $\gamma$-ray transition from $847 \mathrm{keV} 2^{+}$state to the $0^{+}$ground state, as well as a fixed number of $\gamma$-ray multiplicities.

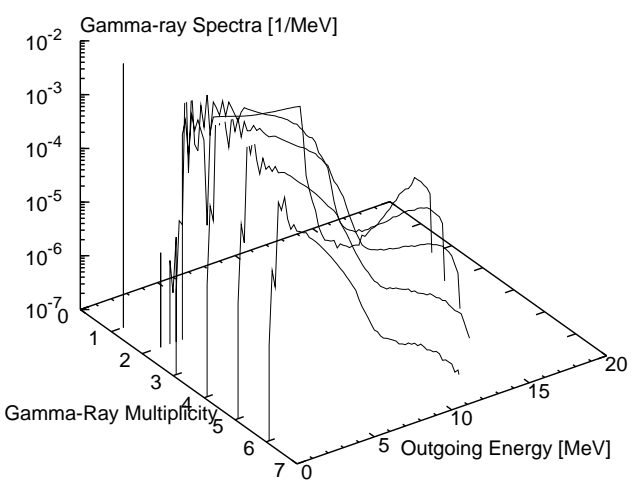

Fig. 5. $\gamma$-ray emission spectra from the neutron inelastic scattering process, for various $\gamma$-ray multiplicities, as a coincidence with the $2^{+} \rightarrow 0+$ transition. Same reaction as Fig. 4 .

that only one neutron is allowed to be emitted, while multiple $\gamma$-rays can be emitted in one simulation. The energy spectra of inelastically scattered neutrons that produce the $847 \mathrm{keV} \gamma$-ray are shown in Fig. 4, for various $m$ values. The $\gamma$-ray spectra, each corresponding to the neutron spectrum case in Fig 4, are shown in Fig. 5.

The calculated average multiplicity is $\bar{m}=3.04$. This implies that a two-step $\gamma$-ray cascading plus E2 transition from the $2^{+}$state after neutron emission is the most probable process. Assuming E1 is the dominant $\gamma$-ray transition, which changes a parity at each decay step, the initial compound nucleus state has an even parity, and the intermediate state (after the first $\gamma$-ray emission) has a negative parity. This $\gamma$-ray cascading might be sensitive to a parity distribution of the compound nucleus. 


\section{Conclusion}

We performed Monte Carlo simulations for particle and $\gamma$ ray emissions from a compound nucleus to evaluate exclusive particle emission spectra and $\gamma$-ray production probabilities for individual reaction processes. Calculated results for the neutron induced nuclear reactions on ${ }^{56} \mathrm{Fe}$ were shown as examples. The results were compared with a traditional deterministic method and it was shown that the results are almost identical. To demonstrate the unique advantages of the Monte Carlo method, a neutron and $\gamma$ ray emission correlation was examined by gating on the $847 \mathrm{keV} \gamma$-ray that is produced by an inelastic scattering process. We reconstructed the partial $\gamma$-ray energy spectra for different $\gamma$-ray multiplicities using the Monte Carlo method.

\section{Acknowledgement}

This work was carried out under the auspices of the National Nuclear Security Administration of the U.S. Department of Energy at Los Alamos National Laboratory under Contract No. DE-AC52-06NA25396.

\section{References}

1. P. G. Young, E. D. Arthur, M. B. Chadwick, Comprehensive nuclear model calculations: Theory and use of the GNASH code, Proc. of the IAEA Workshop on Nuclear Reaction Data and Nuclear Reactors - Physics, Design, and Safety, Singapore: World Scientific Publishing, Ltd., for Trieste, Italy, April 15 - May 17, 1996. Eds. A. Gandini and G. Reffo, pp. 227-404.

2. R. E. MacFarlane, D. G. Foster, Jr., J. Nucl. Mat. 123, (1984) 1047.

3. D. Dashdorj, T. Kawano, P. E. Garrett, J. A. Becker, U. Agvaanluvsan, L. A. Bernstein, M. B. Chadwick, M. Devlin, N. Fotiades, G. E. Mitchell, R. O. Nelson, W. Younes, Phys. Rev. C 75, (2007) 054612.

4. W. Hauser, H. Feshbach, Phys. Rev. 87, (1952) 366.

5. S. Lemaire, P. Talou, T.Kawano, M. B. Chadwick, D. G. Madland, Phys. Rev. C 72, (2005) 024601.

6. S. Lemaire, P. Talou, T. Kawano, M. B. Chadwick, D. G. Madland, Phys. Rev. C 73, (2006) 014602.

7. P. Talou, "Influence of fission modes on prompt neutron characteristics in the neutron-induced fission of ${ }^{235} \mathrm{U}$," International Conference on Nuclear Data for Science and Technology, April 22-27, 2007, Nice, France, editors O. Bersillon, F. Gunsing, E. Bauge, R. Jacqmin, and S. Leray, EDP Sciences, 2008, pp. 317 - 320.

8. J. Randrup, R. Vogt, Phys. Rev. C 80, (2009) 024601.

9. T. Kawano, "CoH: The Hauser-Feshbach-Moldauer statistical model with the coupled-channels theory,'[unpublished]; T. Kawano, S. Chiba, T. Maruyama, Y. Utsuno, H. Koura, A. Seki, "Evaluation of Neutron Reaction Cross Sections for Astrophysics," Proc. of the 2003 Symposium on Nuclear Data, 27-28 Nov., 2003, JAERI, Tokai, Japan, JAERI-Conf 2004-005, (2004) p.196. 